\title{
The Development of Centella asiatica Extract-Loaded BSA Nanoparticles Production to Improve Bioavailability
}

\author{
KITTIYA KESORNBUAKAO ${ }^{1}$ and PATCHANEE YASURIN ${ }^{2 *}$ \\ ${ }^{1}$ Food Technology Department, ${ }^{2}$ Food Biotechnology Program, \\ Biotechnology Faculty, Assumption University, Bangkok, 10240, Thailand. \\ ${ }^{*}$ Corresponding author E-mail: patchaneeYsr@au.edu
}

http://dx.doi.org/10.13005/ojc/320513

(Received: June 27, 2016; Accepted: September 17, 2016)

\begin{abstract}
Centella asiatica (Bao-bog, Pennywort, Gotu kola) is used as a traditional drug widely in Asia. C. asiatica crude extracts showed excellent potential in-vitro but less or no in-vivo activity due to their poor lipid solubility or improper molecular size or both, resulting in poor absorption, poor dosing and poor bioavailability. The Bovine serum albumin (BSA) can attract macromolecular and carry wide variety of molecule. So this research was aimed to develop C. asiatica extract-loaded BSA nanoparticles (CBNP) to improve bioavailability. CBNP was prepared by the desolvation method using three different ratio $C$. asiatica crude chloroform extracts: BSA (1:2, 1:3, and 1:4). The well agar diffusion method was used for evaluating antibacterial activity of CBNP with different concentration $(100,200$, and $300 \mu \mathrm{g} / \mathrm{ml}$ ) against five food borne pathogens (Escherichia coli ATCC25822, Salmonella enterica Typhimurium U302 (DT104b), S. enterica Enteritidis (human), S. enterica 4,5,12:i:- (human) US clone, and Bacillus cereus). The results showed that the antibacterial activity of CBNP did not show significant different on three different ratio and concentration in all food borne pathogens except $S$. enterica Enteritidis (human) and B. cereus $(P<0.05)$. The highest antibacterial active of CBNP was $1.07 \pm 0.46 \mathrm{~cm}$ against $S$. enterica Enteritidis (human) on ratio 1:4, $200 \mu \mathrm{g} / \mathrm{ml}$. The antibacterial activity of CBNP gave almost 2 times higher than free crude $C$. asiatica chloroform extracts. The modified Folin-Ciocalteu method, Ferric reducing antioxidant potential assay and DPPH assay were used for evaluating antioxidant activity. The highest antioxidant activity represented by the amount of phenolic content of CBNP was $14.59 \pm 6.74 \mu \mathrm{gGAE} / \mathrm{mg}$ using ratio 1:2. The amount of phenolic content of CBNP did not show significant different between ratio $1: 2$ and others $(P<0.05)$. For Ferric reducing antioxidant potential assay and DPPH assay, crude chloroform extract showed significantly higher activity than CBNP were $1.00 \pm 0.15 \mathrm{mmol} \mathrm{Fe}{ }^{2+} / \mathrm{mg}$ and $29.44 \pm 8.20 \% \mathrm{DPPH}$ radical scavenging respectively $(P<0.05)$. The entrapment efficiency and loading efficiency of CBNP showed highest value on ratio $1: 4$ which were $96.94 \pm 1.48 \%$ and $42.05 \pm 5.68 \%$ respectively. It's also showed highest in release kinetic in Vitro approximately 12\% during the whole period of 6 hours in both artificial gastric and intestinal juice. C. asiatica crude chloroform extract have higher solubility in water than CBNP but CBNP have higher stability in releasing crude chloroform extract. The results indicated that CBNP showed the promising to increase bioavailability of $C$. asiatica. The statistical analysis was done by ANOVA using SAS software version 9.3.
\end{abstract}

Keywords: Centella asiatica, Nanoparticles, Bioavailability, Antibacterial, Antioxidant. 


\section{INTRODUCTION}

C. asiatica (Bao-bog) is herbal in Asia. In Ayurvedic, an Indian system of medicine, C. asiatica is used for the treatment of leprosy, insanity, asthma, ulcers, eczema, skin and gastrointestinal disorder, arthritis, varicose vein and high blood pressure'. The major biologically active compounds of C. asiatica extract are monoterpenes, sesquiterpene, and triterpenoids ${ }^{2}$. It was found that the chemical complexity of the herbal extracts seem to be important for the bioavailability. Even though, C. asiatica nanopowder gave higher yield of active compound (Asiatic acid) than natural $C$. asiatica powder ${ }^{3}$ there have been some limitations in $C$. asiatica crude extracts that it showed their extra ordinary potential in-vitro but less or no in-vivo activity due to their poor lipid solubility and improper molecular size, resulting in poor absorption, slow delivery, poor dosing and poor bioavailability. Especially, it is also difficult for water-soluble biological active compounds to enter through cell membrane of both human and pathogenic microorganism, which has hydrophobic characteristics. Albumin is a protein that can be obtained from a variety of sources, including egg white (ovalbumin), bovine serum albumin (BSA), and human serum albumin (HSA). Albumin is the major soluble protein of the circulating system and involved in the maintenance of osmotic pressure and binding and transport of nutrients to the cells. Albumin can soluble in water and diluted salt solution very well ${ }^{4}$. The high solubility of albumin (up to $40 \%$ $\mathrm{w} / \mathrm{v})$. Albumin is stable in the $\mathrm{pH}$ range of 4 to 9 and can be heated at $60^{\circ} \mathrm{C}$ up to 10 hours without any deleterious effects and at $\mathrm{pH} 7.4$ makes it an attractive macromolecular carrier capable of accommodating a wide variety of molecule ${ }^{5}$. The development of CBNP are the guild line to overcome these problems. Not only improve drug delivery system but the CBNP also reduce cost from purification steps, and can be applied to use in high valued industry products. Therefore, the objective of this research is to develop CBNP on antibacterial activity, antioxidant activity, and nanoparticles properties.

\section{MATERIALS AND METHODS}

\section{Preparation of sample}

C. asiatica was purchased from local markets in Bangkok, Thailand. The aerial part of $C$. asiatica was used. Fresh C. asiatica were washed with tap water and cut into small pieces. Then it was air dried in oven (Memmert UM500) at $45^{\circ} \mathrm{C}$. The dried samples was finely ground into powder. The powder were kept at $4^{\circ} \mathrm{C}$ before used. ${ }^{2}$

\section{Preparation of C. asiatica crude chloroform extract \\ C. asiatica was extracted with chloroform} using 1:10 ratio $(\mathrm{g} / \mathrm{ml})$. The mixture was macerated at room temperature, $120 \mathrm{rpm}$, for 48 hours and then it was filtered using whatman filter paper no.4. The crude extract was concentrated using rotary evaporators at $45^{\circ} \mathrm{C}$ (BUCHI Rotavapor R-205) are then it was kept at $20^{\circ} \mathrm{C}$ before use. ${ }^{2}$ The $C$. asiatica crude chloroform extracts was further used for preparation of CBNP.

\section{Preparation of C. asiatica extract-loaded BSA nanoparticles}

CBNP was prepared by the desolvation method $^{6}$. The $100 \mathrm{mg}$ of BSA were dissolved in $1 \mathrm{ml}$ of sodium chloride solution $(10 \mathrm{mM})$. Then, $8.0 \mathrm{ml}$ of ethanol was added dropwise into the BSA solution under magnetic stirring $(400 \mathrm{rpm})$ at room temperature. Subsequently, the as-prepared BSA nanoparticles were cross-linked with $0.2 \%$ glutaraldehyde (GA). Then, C. asiatica crude chloroform extract was added into the solution for 24 hours at different ratio of $C$. asiatica to BSA $(1: 2,1: 3$, and 1:4) in the preparation of CBNP. The particles were centrifuged and washed with distilled water. The centrifuged particles were resuspended and disperse in $2 \%$ mannitol, then freeze-dried for 24 hours. The dried nanopowder were kept at room temperature before use.

\section{Antimicrobial activity}

The modified agar well diffusion method ${ }^{2}$ is used. The $100 \mu \mathrm{l}$ of bacteria (approximately $1.5 \times 10^{8} \mathrm{CFU} / \mathrm{ml}$ ) is swab on Mueller-Hinton agar (MHA) plate. The $50 \mu \mathrm{l}$ of $C$. asiatica crude chloroform extract and CBNP at concentration 100, 200, and $300 \mu \mathrm{g} / \mathrm{ml}$ diluted with distilled water were used to test antibacterial activity against Escherichia coli ATCC25822, Salmonella enterica Typhimurium U302 (DT104b), S. enterica Enteritidis (human), S. enterica 4,5,12:i:- (human) US clone, and Bacillus cereus. The $20 \mu \mathrm{l}$ of $50 \mathrm{mg} / \mathrm{ml}$ penicillin $\mathrm{G}$ was used as positive control. The inhibition zones were measured 
to determine the effectiveness of the $C$. asiatica crude extract and CBNP against each bacterium. The experiment was done in duplicate and three replication independently.

\section{Antioxidant activity by Total phenolic content}

The modified Folin-Ciocalteu method ${ }^{7}$ was used for total phenolic content determination of $C$. asiatica crude chloroform extract and CBNP. The $20 \mu \mathrm{l}$ of $10 \mathrm{mg} / \mathrm{ml}$ C. asiatica crude chloroform extract and CBNP was added to $1.58 \mathrm{ml}$ distilled water and $100 \mu \mathrm{l}$ Folin-Ciocalteu phenol reagent. The mixture was then allow to stand for 8 minutes 30 seconds and $300 \mu \mathrm{l}$ saturated sodium carbonate solution was added to the mixture. Then the mixture was incubated without light at room temperature for 30 minutes and observed optical density (OD) at $765 \mathrm{~nm}$. The result were expressed as microgram garlic acid equivalent ( $\mu \mathrm{gGAE} / \mathrm{ml})$. The experiment was done in triplicate and three replication independently.

\section{Antioxidant activity by Ferric reducing antioxidant potential assay (FRAP)}

The modified ferric reducing antioxidant potential assay ${ }^{8}$ was used to determine FRAP value of $C$. asiatica crude chloroform extract and CBNP. The FRAP reagent was prepared using 300 mmol sodium acetate buffer at $\mathrm{pH} 3.6,20 \mathrm{mmol}$ iron chloride and $10 \mathrm{mmol}$ 2,4,6-tripyridyl-s-triazine dissolved in $40 \mathrm{mmol}$ hydrochloric acid at a ratio of 10:1:1 (v:v:v). The reagent was incubated at $37^{\circ} \mathrm{C}$ for 10 minutes before use. The $20 \mu \mathrm{l}$ of $1 \mathrm{mg} / \mathrm{ml}$ the extract and CBNP was added, followed by adding 1000il of FRAP reagent vigorously and kept in the dark for 30 minutes. The optical density (OD) of this mixture was measured at $593 \mathrm{~nm}$. FRAP values were expressed as $\mathrm{mmol} \mathrm{Fe}^{2+} / \mathrm{mg}$ of sample. All measurements were done in triplicate and three replications independently.

\section{Antioxidant activity by DPPH radical scavenging activity}

The modified DPPH radical scavenging activity ${ }^{9}$ was used for percentage $\mathrm{DPPH}$ radical scavenging determination. The $100 \mu \mathrm{l}$ of $1 \mathrm{mg} / \mathrm{ml}$ C. asiatica crude chloroform extract and CBNP were mixed with $3.9 \mathrm{ml}$ DPPH reagent $(50 \mu \mathrm{M})$. The mixture was shaken vigorously and allowed to stand at room temperature in the dark for 30 minutes. The optical density (OD) was measured at $517 \mathrm{~nm}$. The results were expressed as percentage reduction of Percentage reduction of DPPH $=100\left(\frac{A_{0}-A_{c}}{A_{0}}\right)$

Where $A_{0}$ is the initial absorbance and $A_{c}$ is the value for added sample concentration $\mathrm{c}$. All measurements were done in triplicate and three replications independently.

\section{Entrapment efficiency and loading efficiency \\ C. asiatica crude chloroform extract was run} absorbance spectrum to find the best the wavelength ( $\lambda$ max) at which the absorbance is the greatest by UV-vis spectrophotometer. The $2 \mathrm{mg}$ CBNP were dissolved in $1 \mathrm{ml}$ methanol and gently shaken for 24 hours at $37^{\circ} \mathrm{C}$ to completely extract $C$. asiatica crude chloroform extract to methanol ${ }^{11}$. Then the solutions were centrifuged at $12000 \mathrm{rpm}$ for 10 min, and the supernatant was kept and measured optical density (OD) by a UV-vis spectrophotometer at $\lambda$ max. The amount of $C$. asiatica crude chloroform extract entrapped and loaded in CBNP is express as entrapment efficiency and loading efficiency calculated as follows ${ }^{11}$ :

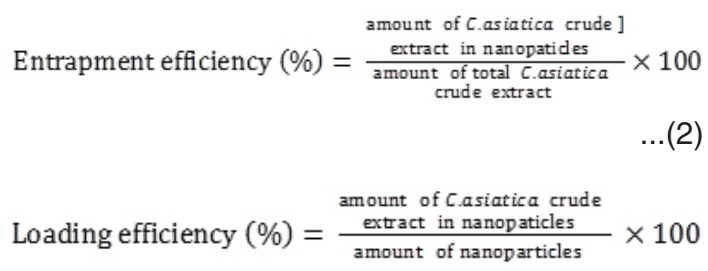

All measurements were done in triplicate and three replications independently.

\section{Solubility and stability}

To compare the solubility of $C$. asiatica crude chloroform extract before and following the encapsulation process, saturation solubility is determined ${ }^{11}$. Excessive samples ( $C$. asiatica crude chloroform extract and CBNP) were dispersed into $1 \mathrm{~mL}$ water and at $200 \mathrm{rpm}, 37^{\circ} \mathrm{C}$. After 24 hours, samples were taken out and filtered through a $0.22 \mu \mathrm{m}$ Millipore membrane. Filtrate was diluted appropriately, and the optical density (OD) were measured by a UV-vis spectrophotometer at $\lambda$ max.

The $1 \mathrm{mg} / \mathrm{mL}$ of CBNP in phosphate buffer solution $(0.01 \mathrm{M}, \mathrm{pH} 7.4)$ were incubated at $200 \mathrm{rpm}$, $37^{\circ} \mathrm{C}$, for 24 hours. At designated time points $(0,0.5$, 
$1,2,3,4,5,6$ hours), the mixture was sampled and the optical density (OD) was measured by UV-vis spectrophotometer at $\lambda \max ^{11}$. The stability of CBNP is calculated as follows:

Percentage reduction of . asiatica $=100\left(\frac{C_{0}-C_{t}}{C_{0}}\right)$

Where $\mathrm{C}_{0}$ is the initial absorbance and $\mathrm{C}_{\mathrm{t}}$ is the absorbance of the sample at time point. All measurements were done in triplicate and three replications independently.

\section{Release kinetic in Vitro}

Release kinetic ${ }^{11}$ methodology was modified. The release of $C$. asiatica crude chloroform extract from CBNP was done by dissolving $20 \mathrm{mg}$ of CBNP in $15 \mathrm{ml}$ artificial gastric juice (0.01 M PBS $\mathrm{pH} 2.0)$ and intestinal juice without enzymes $(0.01 \mathrm{M}$ PBS pH 7.4). The mixture is incubated at $37^{\circ} \mathrm{C}$ at 200 rpm. At designated time points $(0,0.5,1,2,3,4,5,6$ hours), mixture is sampled and centrifuged at 3000 rpm for $10 \mathrm{~min}$. The pellet is resuspended in $100 \mu \mathrm{L}$ of methanol to determine the amount of $C$. asiatica crude chloroform extract released by measuring optical density (OD) by UV-vis spectrophotometer at $\lambda \max$. All measurements were done in triplicate and three replications independently.

\section{Statistical analysis and Experimental design}

All experiments were conducted in three replications and statistical analysis was accomplished using ANOVA with Duncan's multiple range tests $(p<0.05)$ by SAS software version 9.3.

\section{RESULTS AND DISCUSSION}

\section{Antimicrobial activity}

CBNP were prepare by a desolvation method $^{6}$. Nanoparticles are taken up by cells more efficiently than larger micromolecules and therefore, could be used as effective transport and delivery systems. The strategy of applying nanotechnology to plant extracts has been widely used, because nanostructure systems could increase effect of action of plant extracts, promote sustained release of active constituents, reduce the required dose, decrease side effects, and improve activity ${ }^{12,13}$. There are three different of the nanoparticles preparative. The different between these three nanoparticles were ratio of $C$. asiatica crude chloroform extracts to BSA 1:2, 1:3, and 1:4. The well agar diffusion method $^{2}$ was used for evaluating antibacterial activity of CBNP and $C$. asiatica crude chloroform extracts with different concentration $(100,200$, and $300 \mu \mathrm{g} /$ $\mathrm{ml}$ ) against five food borne pathogens (Escherichia coli ATCC25822, Salmonella enterica Typhimurium U302 (DT104b), S. enterica Enteritidis (human), S. enterica 4,5,12:i:- (human) US clone, and Bacillus cereus).

The results of CBNP and $C$. asiatica crude chloroform extracts antibacterial activity were interpreted by using Randomized Complete Block Design (RCBD) with Duncan's multiple range tests in SAS program version9.3 as showed in table1. It was found that antibacterial activities trend of CBNP increased from $C$. asiatica crude chloroform extracts about 2-3 times significantly $(p<0.05)$. C. asiatica crude chloroform extracts have low antibacterial activity because there is a hydrophilic active compound so, it is difficult for water-soluble biological active compounds to permeate through cell membrane, which has hydrophobic characteristics. But, bovine serum albumin (BSA) of CBNP is the protein can attract macromolecular and carry variety of molecule of active compound ${ }^{5}$.Albumin nanoparticles are formed by linkage of amino acid by electrostatic and attraction and covalent linkage reagent (e.g. glutaraldehyde, genipin) and can be prepare to a size of between $100-200 \mathrm{~nm}^{14}$. It also can readily bind and release small molecule ${ }^{5}$. All of these reasons made it can increase an efficiency of absorption to the cells. Moreover, CBNP tend to have more significantly effect on gram-negative bacteria (E. coli ATCC25822, S. enterica Typhimurium U302 (DT104b), S. enterica Enteritidis (human), and S. enterica 4, 5, 12: i: - (human) US clone) than grampositive bacteria (B. cereus). Gram-positive and gram-negative bacteria have difference structural in cell wall composition ${ }^{15}$. In Gram positive bacteria, there is an inner membrane which is surrounded by a thick cell wall made of peptidoglycan ${ }^{16}$. In Gram negative bacteria, there is an inner membrane followed by a thin peptidoglycan layer and an outer membrane ${ }^{16}$. Furthermore, it was found that the different ratio of C. asiatica crude chloroform extracts: BSA and concentration showed no significant difference in antibacterial activity of CBNP against all pathogens except $S$. enterica Enteritidis (human) 
and $B$. cereus $(P<0.05)$. The highest antibacterial active was $1.07 \pm 0.46 \mathrm{~cm}$ using CBNP ration $1: 4$, $200 \mu \mathrm{g} / \mathrm{ml}$ against $S$. enterica Enteritidis (human). The encapsulation process of CBNP can improve antibacterial activity of $C$. asiatica crude chloroform extracts against the pathogens.

\section{Antioxidant activity}

The antioxidant activity can be measured by the ability of the compound that can catch free radicals compounds by scavenging or trapping methods ${ }^{17}$. Herb and spice are rich in phenolic compounds ${ }^{18}$ which are an active compound that have antioxidant properties as protective agent against free radical compound ${ }^{19}$. The major biologically active compounds of $\boldsymbol{C}$. asiatica extract are monoterpenes, sesquiterpene, triterpenoids ${ }^{2}$, polyphenols ${ }^{20}$ and triterpenes ${ }^{21}$. In addition to terpenoids, it also contains high total phenolic contents which contributed by the flavonoids such as quercetin, kaempherol, catechin, rutin, apigenin and naringin and volatile oils such as caryophyllene, farnesol and elemene ${ }^{20,22}$. The antioxidant activity of $C$.asiatica may be due to the reduction of hydroperoxides, inactivation of free radicals, chelation of metal ions or combinations ${ }^{20}$. The antioxidants present in the $C$. asiatica may have different functional properties, such as reactive oxygen species scavenging (quercetin and

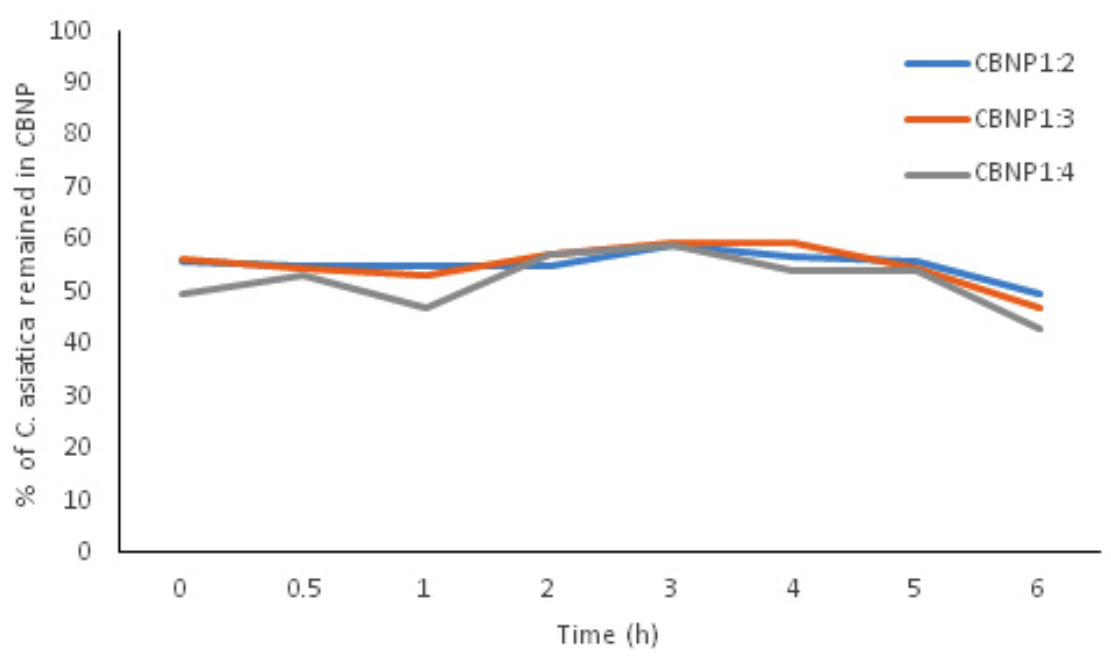

Fig. 1: Stability of CBNP in PBS (pH 7.4) at $37^{\circ} \mathrm{C}$ over period of 6 hours

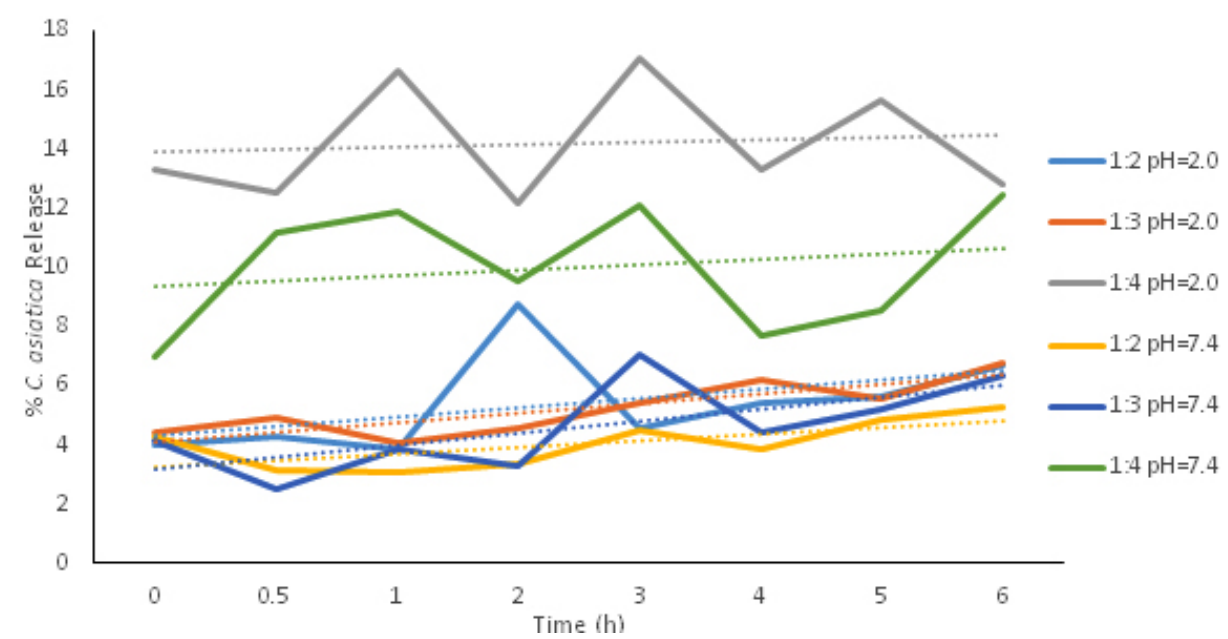

Fig. 2: Release rate of $C$. asiatica from CBNP in vitro in PBS (in artificial gastric juice at $\mathrm{pH} 2.0$ and artificial intestinal juice at $\mathrm{pH} 7.4$ ) at $37^{\circ} \mathrm{C}$ over period of 6 hours 


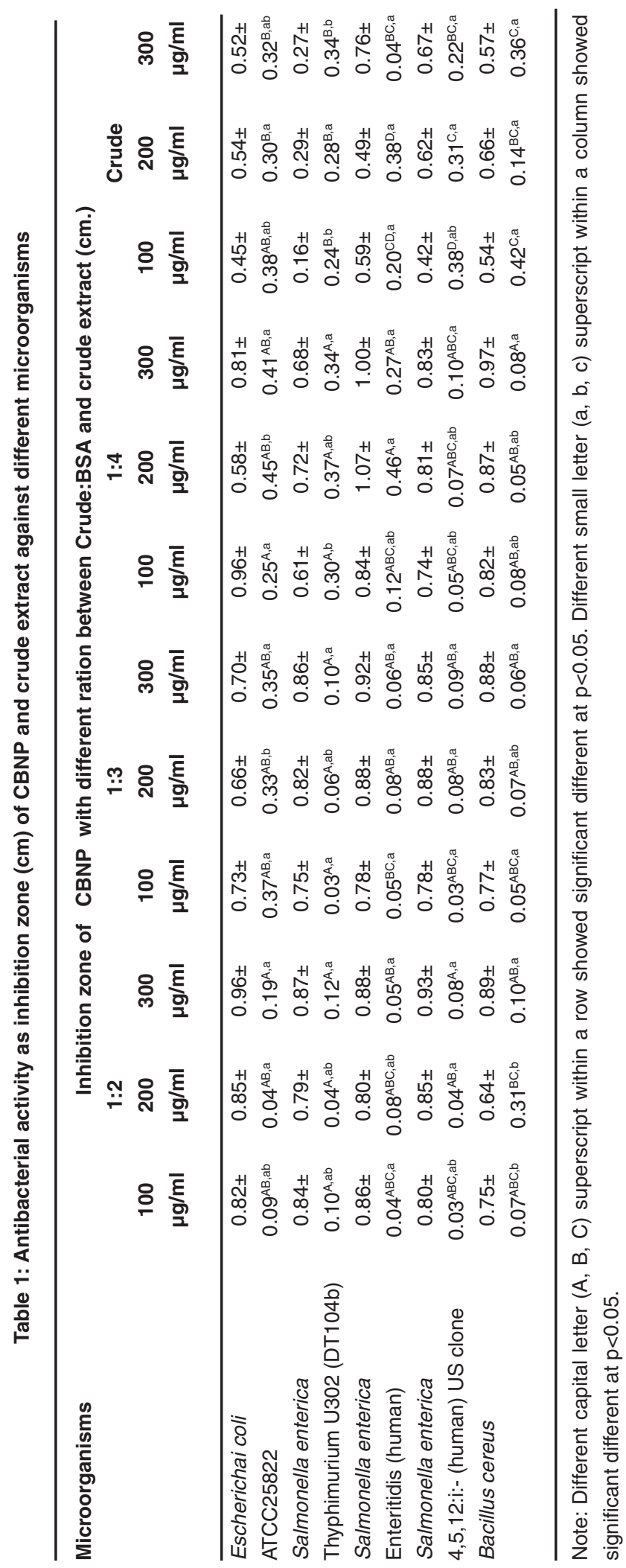


catechins) ${ }^{23}$, inhibition of the generation of free radicals and chain-breaking activity, e.g.p-coumaric acids $^{24}$ and metalchelation ${ }^{25}$.

The modified Folin-Ciocalteu method ${ }^{7}$, Ferric reducing antioxidant potential assay ${ }^{8}$ and DPPH radical scavenging ${ }^{9}$ were used for evaluating antioxidant activity of CBNP and $C$. asiatica crude chloroform extracts. The results were interpreted by using Randomized Complete Block Design (RCBD) with Duncan's multiple range tests in SAS program version9.3 as showed in table2. The phenolic compound was bind to BSA mainly by hydrogen bond, electrostatic, and hydrophobic interaction. Molecular structure and the number of hydroxyl group of phenolic compound are also the factor that effect phenolic compound binding with $\mathrm{BSA}^{26}$. Total phenolic content of CBNP and crude extract were determined in comparison with standard garlic acid and the results were expressed in terms of $\mu \mathrm{g}$ GAE/ $\mathrm{mg}$ dried weight. The highest antioxidant activity represented by the amount of phenolic content of
CBNP was $14.59 \pm 6.74 \mu \mathrm{gGAE} / \mathrm{mg}$ using ratio $1: 2$. The amount of phenolic content of CBNP did not show significant different between ratio 1:2 and others $(P<0.05)$. For Ferric reducing antioxidant potential assay and DPPH assay, crude chloroform extract showed significantly higher activity than CBNP were $1.00 \pm 0.15 \mathrm{mmol} \mathrm{Fe} \mathrm{Fe}^{2+} / \mathrm{mg}$ and 29.44 $\pm 8.20 \% \mathrm{DPPH}$ radical scavenging respectively $(P<0.05)$. After process of nanoparticles, C.asiatica loss of its activity in ferric reducing antioxidant potential assay and DPPH radical scavenging. Because of the active compound maybe are already bind with protein and changed the structural to be inactive form.

\section{Entrapment efficiency and loading efficiency}

The entrapment efficiency and loading efficiency were calculated using equations 2 and 3 mentioned above. The results were interpreted by using Randomized Complete Block Design (RCBD) with Duncan's multiple range tests in SAS program version9.3 as showed in table3. CBNP using ratio

Table 2: Total phenolic content, Ferric reducing antioxidant potential, and DPPH radical scavenging of CBNP and $C$. asiatica crude chloroform extract

\begin{tabular}{lccc}
\hline $\begin{array}{l}\text { CBNP with different ratio } \\
\text { between Crude: } \\
\text { BSA and crude }\end{array}$ & $\begin{array}{c}\text { Total phenolic content } \\
\text { ( } \mu \mathrm{g} \mathrm{GAE} / \mathrm{mg} \text { dried weight) }\end{array}$ & $\begin{array}{c}\text { FRAP } \\
\text { (mmol Fe } \text { (m) }^{2+} \\
\text { dried weight) }\end{array}$ & $\begin{array}{c}\text { DPPH } \\
\text { (\% reduction } \\
\text { of DPPH) }\end{array}$ \\
\hline $1: 2$ & $14.59 \pm 6.74^{\mathrm{A}}$ & $0.53 \pm 0.16^{\mathrm{B}}$ & $11.55 \pm 4.70^{\mathrm{B}}$ \\
$1: 3$ & $13.15 \pm 5.94^{\mathrm{A}}$ & $0.49 \pm 0.06^{\mathrm{B}}$ & $13.57 \pm 8.99^{\mathrm{B}}$ \\
$1: 4$ & $13.15 \pm 7.13^{\mathrm{A}}$ & $0.50 \pm 0.01^{\mathrm{B}}$ & $5.76 \pm 10.44^{\mathrm{B}}$ \\
crude & $13.15 \pm 1.62^{\mathrm{A}}$ & $1.00 \pm 0.15^{\mathrm{A}}$ & $29.44 \pm 8.20^{\mathrm{A}}$ \\
\hline
\end{tabular}

Note: Different superscript within a column showed significant different at $p<0.05$

Table 3: Entrapment efficientcy, loading efficiency, and solubility of CBNP and C. asiatica crude chloroform extract

\begin{tabular}{lccc}
\hline $\begin{array}{l}\text { CBNP with different } \\
\text { ratio between Crude: } \\
\text { BSA and crude }\end{array}$ & $\begin{array}{c}\text { Entrapment } \\
\text { efficiency(\%) }\end{array}$ & $\begin{array}{c}\text { Loading } \\
\text { Efficiency(\%) }\end{array}$ & $\begin{array}{c}\text { Solubility } \\
(\boldsymbol{\mu g} / \mathbf{m l})\end{array}$ \\
\hline $1: 2$ & $42.77 \pm 4.23^{\mathrm{B}}$ & $41.28 \pm 8.91^{\mathrm{A}}$ & $203.58 \pm 113.81^{\mathrm{A}}$ \\
$1: 3$ & $59.48 \pm 8.90^{\mathrm{B}}$ & $36.60 \pm 7.56^{\mathrm{A}}$ & $172.59 \pm 13.53^{\mathrm{A}}$ \\
$1: 4$ & $96.94 \pm 1.48^{\mathrm{A}}$ & $42.05 \pm 5.68^{\mathrm{A}}$ & $168.86 \pm 78.78^{\mathrm{A}}$ \\
crude & & & $244.34 \pm 17.10^{\mathrm{A}}$ \\
\hline
\end{tabular}

Note: Different superscript within a column showed significant different at $p<0.05$ 
1:4 has entrapment efficiency significantly higher than ratio $1: 3$ and $1: 2$ which were $96.94 \pm 1.48 \%$, $59.48 \pm 8.90 \%$, and $42.77 \pm 4.23 \%$ respectively $(p<0.05)$. There are no significantly difference in entrapment efficiency between CBNP using ratio 1:3 and 1:2 $(p<0.05)$. For loading efficiency, there are no significantly different among ratio of CBNP $(p<0.05)$. CBNP using ratio 1:4 also has highest percentage followed by $1: 2$ and $1: 3$ which were $42.05 \pm 5.68 \%$, $41.28 \pm 8.91 \%$, and $36.60 \pm 7.56 \%$ respectively.

\section{Solubility and Stability}

To determine the solubility of $C$. asiatica crude chloroform extracts before and following the encapsulation process, $C$. asiatica crude chloroform extracts and CBNP were dissolved in water. The results were interpreted by using Randomized Complete Block Design (RCBD) with Duncan's multiple range tests in SAS program version9.3 as showed in table3. The result showed that $C$. asiatica crude chloroform extracts has highest solubility in water which is $244.34 \pm 17.10 \mu \mathrm{g} / \mathrm{ml}$. But, there are no significant difference between $C$. asiatica crude chloroform extracts with others CBNP $(p<0.05)$. While, CBNP using ratio 1:4 has lowest solubility which is $168.86 \pm 78.78 \mu \mathrm{g} / \mathrm{ml}$. The process of making CBNP improve hydrophobic capacity of crude extract. It is difficult for water-soluble biological active compounds to enter through cell membrane of both human and pathogenic microorganism, which has hydrophobic characteristics. So, improving of hydrophobic capacity of crude extract make it can be better in entering through the cell.

To study the stability of CBNP, it was incubated in PBS (0.01M, pH 7.4) and calculate their concentration with time by equation 4. CBNP showed very stable under the condition over period of 6 hours as showed in figures 1 . The nanoparticles process made the stability of $\mathrm{C}$. asiatica crude chloroform extracts by protecting it from hydrolysis and biotransformation ${ }^{11}$. CBNP provided stability of the $C$. asiatica crude extract so, it can be used as process to prolong shelf-life of plant extract.

\section{Release Kinetic in vitro}

In the study of release kinetic in vitro, artificial gastric juice (0.01 M PBS $\mathrm{pH} 2.0)$ and artificial intestinal juice (0.01 M PBS pH 7.4) were used to imitate the environment in stomach and intestine. The cumulative percentage of released C. asiatica were determine as showed in figure2. The release rate of $C$. asiatica from CBNP using same ratio in the gastric juice was tend to higher than intestinal juice. CBNP using ratio 1:4 released highest in both artificial gastric juice (0.01 M PBS pH 2.0) and artificial intestinal juice (0.01 M PBS pH 7.4). At $\mathrm{pH} 2.0$ the BSA protein was unfolded and release C. asiatica out from the particles. BSA was most stable at $\mathrm{pH} 7$ but rapidly degraded by aggregation and hydrolysis at $\mathrm{pH} 2^{27}$. CBNP can be release in very low $\mathrm{pH}$ so, it can be apply to be oral medicine ${ }^{28}$.

\section{CONCLUSIONS}

The different ratio of $C$. asiatica to BSA $(1: 2,1: 3$, and 1:4) has effect on bioavailability. CBNP were less solubility in water than crude extract thus, CBNP can improve hydrophobic capacity of C. asaitica crude extract. So, this study is focus on improving ability of $C$. asaitica crude extract in absorption to the cell. Higher hydrophobic capacity of the CBNP makes it can penetrate into cell membrane and enter to the cell. These result is parallel to antibacterial activity of CBNP that showed 2 times higher than crude extract. Moreover, CBNP has no significantly different in total phenolic content compare with crude extract, but has significantly lower in FRAP and DPPH $(p<0.05)$. CBNP also showed stability and releasing of $C$. asiatica in PBS. It can be conclude that CBNP at ratio 1:4 is most effective in an economical way because using less crude extract. The development of CBNP would be a promising improving bioavailability of $C$. asaitica crude extract.

\section{ACKNOWLEDGEMENTS}

This research is supported by the Assumption University (research grant P-58-388). 


\section{REFERENCES}

1. Ariffin, F.; Heong, Chew S.; Bhupinder, K.; Karim, AA.; Huda, N. Antioxidant capacity and phenolic composition of fermented Centella asiatica herbal teas. J Sci Food Agric. 2011, 91, 2731-9

2. Rattanakom, S.; Yasurin, P. Antibacterial activity, antioxidant activity and chemical profiling of Centella asiatica under different extraction solvents. Orient. J. Chem. 2015, 31, 2453-2459

3. Borhan, M. Z.; Nee, T. Y. Synthesis of $\mathrm{TiO}_{2}$ nanopowders from red gypsum using EDTA as complexing agent. Journal of Nanostructure in Chemistry. 2014

4. Lohcharoenkal, Warangkana.; Wang, Liying.; Chen, Yi Charlie.; Rojanasakul, Yon. Protein Nanoparticles as Drug Delivery Carriers for Cancer Therapy. BioMed Research International. 2014

5. Kratz, F. Albumin as a drug carrier: design of prodrugs, drug conjugates and nanoparticles. PubMed. 2008 , 132, 171-83

6. Yu, Z.; Yu, M.; Zhang, Z.; Hong, G.; Xiong, Q. Bovine serum albumin nanoparticles as controlled release carrier for local drug delivery to the inner ear. Nanoscale Research Letters. 2014, 9, 343

7. Ragazzi, E.; Veronese, G. Quantitative analysis of phenolic compound after thin-layer chromatographic separation. J Chromatogr $A$. 1973, 77, 369-75

8. Benzie, IF.; Strain, JJ. Ferric reducing/ antioxidant power assay: Direct measure of total antioxidant activity of biological fluids and modified version for simultaneous measurement of total antioxidant power and ascorbic acid concentration. Methods Enzymol. 1999, 299, 15-27

9. Brand-Williams, W.; Cuvelier, ME.; Berset, C. Use of a free radical method to evaluate antioxidant activity. LWT-Food Science and Technology. 1995, 28, 25-30

10. Molyneux, P.The use of the stable free radical diphenylpicrylhydrazyl (DPPH) for estimating antioxidant activity. Songklanakarin J. Sci. Technol. 2004, 26, 211-219

11. Xie, X.; Tao, Q.; Zou, Y.; Zhang, F.; Guo, M.; Wang, Y.; Wang, H.; Zhou, Q.; Yu,
Shuqin. PLGA Nanoparticles Improve the Oral Bioavailability of Curcumin in Rats: Characterizations and Mechanisms. Journal of Agricultural and Food Chemistry. 2011, 59, 9280-9289

12. V, Ghosh.; S, Saranya.; A, Mukherjee.; N, Chandrasekaran. Antibacterial microemulsion prevents sepsis and triggers healing of wound in wistar rats. Colloids and Surfaces B: Biointerfaces. 2013, 105, 152-157

13. Rajendran, R.; Radhai, R.; Kotresh, TM.; Csiszar, E. Development of antimicrobial cotton fabrics using herb loaded nanoparticles. Carbohydrate Polymers. 2013, 91, 613-617

14. Weber, C. Desolvation process and surface characterisation of protein nanoparticles. International Journal of Pharmaceutics. 2000, 194, 91-102

15. Madigan, M.; Martinko, J. Brock biology of microorganisms. 11th ed. Englewood Cliffs. NJ: Prentice Hall. 2005

16. Brown, Lisa.; M, Julie.; Wolf, Rafael PradosRosales. Arturo Casadevall Through the wall: extracellular vesicles in Gram-positive bacteria, mycobacteria and fungi Nature Reviews Microbiology. 2015, 13, 620-630

17. Huang, D.; Ou, B.; Prior, R.L. The chemistry behind antioxidant capacity assays. J. Agric. Food Chem. 2005, 53, 1841-1856

18. NV, Yanishlieva.; E, Marinova.; J, Pokorny. Natural antioxidant from herb and spices. Eur J Lipid Sci Technol. 2006, 108, 776-93

19. D, de Beer.; E, Joubert.;W.C.A., Gelderblom.; M, Manley. Phenolic Compounds: A Review of Their Possible Role as In Vivo Antioxidants of Wine. 2002, 23

20. MK, Zainol.; A, Abd-Hamid.; S, Yusof.; R, Muse. Antioxidative activity and total phenolic compounds of leaf, root and petiole of four accessions of Centella asiatica(L.) Urban. Food Chemistry. 2003, 81, 575-581

21. Inamdar, P.K.; Yeole, R. D.; Ghogare, A. B.; de Souza, N. J. Determination of Biologically Active Constituents in Cente-lla Asiatica. J. Chromatogr. A. 1996, 742, 127-30

22. Chong, NJ.; Aziz, Z. A Systematic Review of the Efficacy of Centella asiatica for Improvement of the Signs and Symptoms of Chronic Venous 
Insufficiency. EvidencebasedComplementary and Alternative Medicine: eCAM. 2013, 627182.10.1155/627182

23. Hatano ,T.; Edamatsu, R.; Hiramatsu, M.; Moti, A.; Fujita, Y.; Yasuhara, T.; Yoshida, T.; Okuda, T. Effects of tannins and related polyphenols on superoxide anion radical, and on 1, 1-diphenyl-2-picrylhydrazyl radical. Chemical and Pharmaceutical Bulletin.1989, 37, 2016-2021

24. Laranjinha, J.; Vieira, O.; Madeira, V.; Almeida, L. Two related phenolic antioxidants with opposite effects on vitamin $E$ content in low density lipoproteins oxidized by ferrylmyoglobin: consumption vs regeneration. Archives of biochemistry andbiophysics. 1995, 323, 373-381

25. Van Acker, SA.; Van Balen, GP.; Van den Berg, DJ.; Bast, A.; Van der Vijgh, WJ. Influence of iron chelation on the antioxidant activity of flavonoids. Biochemicalpharmacology. 1998, 56, 935-943

26. Tianxi, He.; Qionglin, Liang.; Tingting, Luo.; Yiming, Wang.; Guoan, Luo. Study on Interactions of Phenolic Acid-Like Drug Candidates with Bovine Serum Albumin by Capillary Electrophoresis and Fluorescence Spectroscopy. Journal of Solution Chemistry. 2010, 39, 1653-1664.

27. Tia, Estey.; Jichao, Kang.; Steven P., Schwendeman.; John F., Carpenter. BSA Degradation under Acidic Conditions:A Model for Protein Instability during Release from PLGA Delivery Systems. Journal of pharmaceutical sciences. 2006, 95

28. Yasurin, P.; Sriariyanun, M.; Phusantisampan, T. Review: the bioavailability activity of Centella asiatica. KMUTNB Int J Appl Sci Technol. 2016, 9, 1-9. 